\title{
Stability analysis of a model of atherogenesis: an energy estimate approach II
}

\author{
A.I. Ibragimov ${ }^{\mathrm{a}}$, C.J. McNeal ${ }^{\mathrm{bc}}$, L.R. Ritter ${ }^{\mathrm{d}} *$ and J.R. Walton ${ }^{\mathrm{e}}$ \\ ${ }^{a}$ Department of Mathematics, Texas Tech University, Lubbock, TX 79409, USA; ${ }^{b}$ Division of \\ Cardiology, Department of Internal Medicine, Temple, TX 76508, USA; ${ }^{c}$ Division of Endocrinology, \\ Department of Pediatrics, Scott \& White, Temple, TX 76508, USA; ${ }^{d}$ Department of Mathematics, \\ Southern Polytechnic State University, Marietta, GA 30060, USA; ${ }^{e}$ Department of Mathematics, \\ Texas A \& M University, College Station, TX 77843-3368, USA
}

(Received 21 July 2008; final version received 12 December 2008)

\begin{abstract}
This paper considers modelling atherogenesis, the initiation of atherosclerosis, as an inflammatory instability. Motivated by the disease paradigm articulated by Russell Ross, atherogenesis is viewed as an inflammatory spiral with positive feedback loop involving key cellular and chemical species interacting and reacting within the intimal layer of muscular arteries. The inflammation is modelled through a system of nonlinear reaction-diffusion-convection partial differential equations. The inflammatory spiral is initiated as an instability from a healthy state which is defined to be an equilibrium state devoid of certain key inflammatory markers. Disease initiation is studied through a linear, asymptotic stability analysis of a healthy equilibrium state. Various theorems are proved giving conditions on system parameters guaranteeing stability of the health state and conditions on system parameters leading to instability. Among the questions addressed in the analysis is the possible mitigating effect of antioxidants upon transition to the inflammatory spiral.
\end{abstract}

Keywords: atherosclerosis; atherogenesis; chemotaxis; stability analysis; energy estimate

2000 Mathematics Subject Classification: 35K55; 92C17; 92C50

\section{Introduction}

Mathematical models have a significant role to play in understanding the structure, functioning, evolution and diseases of the cardiovascular system. Moreover, formulating, simulating and analysing such models offer a vast array of challenges. (See [14] for an interesting survey on the subject.) This article is a continuation of a program to develop, analyse and simulate mathematical models of atherosclerosis initiated by the authors in [7].

Atherosclerosis is a very complex chronic disease of the arterial system with many manifestations and many routes to initiation and progression [4,13,15-17]. Biochemical, genetic, mechanical and pathogenic factors conspire to initiate and promote the disease. The focus of [9] and the present contribution is the role played by inflammation in atherogenesis $[5,15,16]$. This is not to suggest that genetic, mechanical and pathogenic factors are unimportant or are subordinate to the inflammatory processes considered herein and in [7] and [9]. Account is taken of them through parameters in the equations studied below that model a particular inflammatory cycle thought to play a fundamental role in

\footnotetext{
*Corresponding author. Email: 1ritter@spsu.edu
} 
atherogenesis. Histology of the arterial wall also plays an important role in atherosclerosis. Histological details depend upon the basic arterial type (elastic arteries vs. muscular arteries), artery location, age as well as disease initiated changes such as adaptation and remodelling (from chronic hypertension, for example) and degradation (inflammation induced medial diminution, for example). In [9] and the present study, attention is focused is upon the inner most layer of the arterial wall, the tunica intima since the very beginning stages of atherosclerosis are largely confined to this layer.

The first extension to the analysis in [7] given by the authors appears in [9] where we used an energy estimate to analyse the stability of a model of atherogenesis that focused on only four species involved in the inflammatory process, and which considered the interplay between viable and apoptotic immune cells. Herein, we consider an extended model that includes the role of low-density lipoproteins (LDL) in both a native and chemically modified state (oxLDL), as well as reactive oxygen species (referred to throughout as 'free radicals') present in the subintimal layer. Anti-oxidant effects are also introduced through a parameter. While these species were considered as part of the original model proposed by the authors in [7], they were ignored in the numerical and analytical studies appearing in [7-9].

The inflammatory process modelled in [7] involved the following ingredients: two cellular species (smooth muscle cells and macrophages), lesion debris (necrotic cells, lipid core of foam cells and smooth muscle cells) ${ }^{1}$ and three molecular species (LDL, chemically modified LDL and a chemical signally species). Each of the cellular and molecular species are to be viewed as representative of large classes of cells or molecules exhibiting the functional response attributed to the respective representative. For example, while a number of immune system cells play a role in the in ammatory processes occurring during atherogenesis, the monocyte derived macrophages are probably the dominant players in the creation of the lipidladen foam cells that collect in the lipid-rich core of atherosclerotic plaques. Hence to simplify the model, macrophages are the only immune systems cells included in the modelling. Similarly, the LDL species should be viewed as a generic representative of a large class of lipid molecules and oxLDL as a generic representative from the corresponding class of lipids that have been oxidized (chemically modified) by free radicals.

The point of view articulated in [16] and motivating the model adopted in [7] is that atherosclerotic plaques form as a consequence of chronic inflammation sustained through a positive inflammatory feedback loop $[5,6,15,16]$. The heart of this disease paradigm consists of the following process elements. Through various means such as shear stress [2], a portion of the endothelial layer of a muscular arterial wall develops a 'leaky' spot permitting accelerated transport of LDL (and other macromolecular species) through the endothelial barrier into the intima where they tend to concentrate due to the difficulty of further passage through the inner elastic lamina into the media [12]. Simultaneously, monocytes also enter the intima in response to chemical signalling from an initiating inflammatory reaction (possibly due to viral or bacterial insult, for example) [10]. The LDL is eventually chemically modified by reactive oxygen species (typically referred to as free radicals) produced through natural metabolic processes occurring in various cellular species within the arterial wall (e.g. smooth muscle cells, endothelial cells, fibroblasts, etc.). Macrophages have an affinity for the oxLDL resulting from this chemical modification process (Indeed, there is a strong experimental evidence that macrophages exhibit positive chemotactic sensitivity to these oxLDL species), eventually becoming foam cells (i.e. macrophages engorged with oxLDL particles). These engorged macrophage-derived foam cells are no longer capable of doing their customary job of removing the debris produced by the inflammatory processes; in fact they become components of the growing lesion debris. The growing lesion debris produces various chemical signalling species that attract additional macrophages to the lesion site 
which then get 'corrupted' by the oxLDL species resulting in a chronic inflammatory spiral $[6,15,16]$. Another aspect of plaque growth modelled in [7] involves the recruitment (via chemical signalling) of smooth muscles to the lesion site and their role in forming a tough cap to isolate the lesion from healthy tissue and the lumen. However, since this is characteristic of the latter stages of the disease process, we do not consider this process at present.

A number of important issues were not addressed in [7] including how to model plaque growth with significant luminal occlusion and how to determine under what conditions the runaway inflammatory/plaque growth spiral occurs and conversely under what conditions the natural defence mechanisms of the body prevent it. The latter question is the subject of [9] and the present paper.

The perspective taken in [9] and extended herein on the latter question is that it is one of stability of the non-linear reaction-diffusion-chemotaxis system used to model the inflammatory processes initiating atherosclerotic plaque growth. More specifically, the question investigated is whether certain equilibrium states of the governing system of nonlinear partial differential equations, referred to as 'healthy states', are linearly, asymptotically stable. These healthy states are characterized by the absence of inflammatory markers, which in the context of the model described above, correspond to equilibrium states in which the macrophage, debris and chemical signal species are at some baseline level in the intimal layer that is commensurate with normal immune function. As stated, the results presented here differ from those obtained in [9] as we account here for LDL, oxLDL and free-radical interaction and reactions. In addition, we consider herein both a closed system - in which boundary transport (into the intima via the endothelial layer) is not allowed - and a more realistic system allowing for boundary transport of some species. For the latter case, the mathematical methods employed in [9] are adapted to account for the increased mathematical complexity introduced.

\section{Mathematical model}

The model for atherogenesis of interest here tracks the evolution of six generic 'species' which are major contributors to the initial stages of atherosclerosis. These species are generic in that they are representative of classes of factors contributing to the inflammatory processes leading to disease initiation. In this spirit, these representative species are given the labels: immune cells (principally macrophages), debris (developing lesion), chemoattractant, native LDL, oxidized LDL and free radicals, and denoted $\mathcal{I}, \mathcal{D}, \mathcal{C}, \mathcal{L}, \mathcal{L}_{\text {ox }}$ and $\mathcal{R}$, respectively.

The governing equations for this simplified model are of the form:

$$
\begin{gathered}
\frac{\partial \mathcal{I}}{\partial t}=\operatorname{div}\left(\mu_{1} \nabla \mathcal{I}\right)-\operatorname{div}(\chi(\mathcal{I}, \mathcal{C}) \nabla \mathcal{C})-d_{11} \mathcal{I}-a_{15} \mathcal{I} \mathcal{L}_{\mathrm{ox}}-a_{12} \mathcal{I D}+M \phi_{0}, \\
\frac{\partial \mathcal{D}}{\partial t}=\operatorname{div}\left(\mu_{2} \nabla \mathcal{D}\right)+c_{15} \mathcal{I} \mathcal{L}_{\mathrm{ox}}-a_{21} \mathcal{I} \mathcal{D}-d_{22} \mathcal{D} \\
\frac{\partial \mathcal{C}}{\partial t}=\operatorname{div}\left(\mu_{3} \nabla \mathcal{C}\right)+p_{32} \mathcal{D}-a_{31} \mathcal{C I}-d_{33} \mathcal{C} \\
\frac{\partial \mathcal{L}}{\partial t}=\operatorname{div}\left(\mu_{4} \nabla \mathcal{L}\right)-a_{46} \mathcal{L} \mathcal{R}+b_{4} A_{\mathrm{ox}} r_{4} \mathcal{L}_{\mathrm{ox}} \\
\frac{\partial \mathcal{L}_{\mathrm{ox}}}{\partial t}=\operatorname{div}\left(\mu_{5} \nabla \mathcal{L}_{\mathrm{ox}}\right)+c_{46} \mathcal{L} \mathcal{R}-A_{\mathrm{ox}} r_{4} \mathcal{L}_{\mathrm{ox}}-b_{15} \mathcal{I} \mathcal{L}_{\mathrm{ox}} \\
\frac{\partial \mathcal{R}}{\partial t}=\operatorname{div}\left(\mu_{6} \nabla \mathcal{R}\right)-b_{46} \mathcal{L} \mathcal{R}-b_{6} A_{\mathrm{ox}} \mathcal{R}+p_{\mathcal{R}}
\end{gathered}
$$


Here, div and $\nabla$ denote the usual divergence and gradient operators. The various terms appearing on the right-hand side of these equations require some discussion.

The term $-\chi(\mathcal{I}, \mathcal{C}) \nabla \mathcal{C}$ in Equation (1) is the portion of immune cell flux due to chemotaxis, and the coefficient $\chi(\mathcal{I}, \mathcal{C})$ is the chemotactic sensitivity. ${ }^{2}$ The term $d_{11} \mathcal{I}$ represents natural turnover of immune cells. The two terms $a_{15} \mathcal{I} \mathcal{L}_{\text {ox }}$ and $a_{12} \mathcal{I D}$ in (1) give the rate at which the macrophage population is diminished through foam cell formation (through binding with oxidized LDL), and through normal immune function. The latter, for example, could be accounted for by viable macrophages binding with debris for eventual processing in the liver. ${ }^{3}$ Finally, in the stability analyses that follow, we will be considering a perturbation off of a constant level of macrophages. In essence, we are looking at a small time window. The term $M \phi_{0}$ in (1) represents a baseline level of immune cells present. In general, $M \phi_{0}$ could depend on the level of chemoattractant, especially at the boundary where transport across the endothelial layer can occur. We can assume that over the time scales of interest, the value is constant. Mass transport through the endothelial layer will be considered in a later section.

The term $b_{15} \mathcal{I} \mathcal{L}_{\text {ox }}$ appearing in Equation (5) represents conversion of oxidized LDL into foam cells. The balance of mass is captured by $c_{15} \mathcal{I} \mathcal{L}_{\mathrm{ox}}$ which appears in Equation (2); thus we have $c_{15}=a_{15}+b_{15}$. The term $a_{21} \mathcal{I D}$ is the rate at which debris is removed by uncorrupted macrophages while $d_{22} \mathcal{D}$ is a natural turnover rate for debris.

In (3), $p_{32} \mathcal{D}$ is the rate at which chemoattractant is produced by the lesion debris, while $a_{31} \mathcal{C I}$ is the rate by which the chemoattractant concentration is diminished by binding with macrophages. The term $d_{33} \mathcal{C}$ is a natural chemical degradation rate for the chemoattractant.

In (4)-(6), $a_{46} \mathcal{L} \mathcal{R}$ and $b_{46} \mathcal{L} \mathcal{R}$ are the rates at which the native LDL and free radical concentrations are diminished by free radical oxidation of the native LDL (and their sum $c_{46}=a_{46}+b_{46}$ added to the $\mathcal{L}_{\text {ox }}$ concentration), while $A_{\text {ox }} r_{4} \mathcal{L}_{\text {ox }}$ is the rate at which the anti-oxidant concentration, $A_{\mathrm{ox}}$, is able to reverse the oxidative damage done to LDL by the free radicals. The coefficient $b_{4}$ (with $0<b_{4}<1$ ) is an efficiency parameter representing the fraction of the products of the $A_{\mathrm{ox}}-L_{\mathrm{ox}}$ reverse reaction feeding back into the native LDL population. ${ }^{4}$ Finally, $p_{\mathcal{R}}$ in (6) is the rate of free radical production, ${ }^{5}$ and $A_{\mathrm{ox}} b_{6} \mathcal{R}$ is the rate at which the anti-oxidant concentration is able to reduce the free radical concentration through direct reaction.

In the next sections, we perform a linear stability analysis of the form in our recent work [9]. We will consider the Equations (1)-(6) to hold in a domain $\Omega$ with inner and outer boundaries $\Gamma_{1}$ and $\Gamma_{2}$, respectively. Though we will not specify the geometry exactly, $\Omega$ can be taken as a deformed annulus in two dimensions, or an annular (deformed) cylinder in three dimensions. In the first section that follows, we will consider Equations (1)-(6) to be coupled with homogeneous Neumann boundary conditions on $\Gamma_{1} \cup \Gamma_{2}$. This will result in natural extension of the method developed in [9] to the larger system considered here. Later, we will modify the method to consider Equations (1)-(6) and allow for a non-homogeneous boundary condition on $\Gamma_{1}$ for immune cells, LDL and the chemoattractant.

Transport of chemoattractant and immune cells - monocytes that are differential into macrophages in the tissue - will be considered as influenced by the differential of the level of chemoattractant at the boundary with some baseline level of chemoattractant in the plasma. If the level of chemoattractant at the endothelium exceeds this baseline, monocytes respond by entering into the intimal layer where they become macrophages. Similarly, chemoattractant exits the intima into the plasma when the baseline level is exceeded. The transport of native LDL, into the arterial wall - or out in the case of reverse transport - will likewise be considered. This will allow us to introduce the baseline level 
of LDL in the plasma which will affect the existence and nature of an equilibrium healthy state. This leads to additional mathematical challenges that are addressed in Section 4.

\section{Stability analysis with homogeneous boundary conditions}

As stated, we consider Equations (1)-(6) to govern the various species within the domain $\Omega$ and impose the homogeneous boundary conditions

$$
\frac{\partial \mathcal{I}}{\partial \mathbf{n}}=\frac{\partial \mathcal{D}}{\partial \mathbf{n}}=\frac{\partial \mathcal{C}}{\partial \mathbf{n}}=\frac{\partial \mathcal{L}}{\partial \mathbf{n}}=\frac{\partial \mathcal{L}_{\mathrm{ox}}}{\partial \mathbf{n}}=\frac{\partial \mathcal{R}}{\partial \mathbf{n}}=0
$$

on $\Gamma_{1} \cup \Gamma_{2}$. We begin by assuming that there is a constant equilibrium state $\left(\mathcal{I}_{\mathrm{e}}, \mathcal{D}_{\mathrm{e}}, \mathcal{C}_{\mathrm{e}}, \mathcal{L}_{\mathrm{e}}, \mathcal{L}_{\text {oxe }}, \mathcal{R}_{\mathrm{e}}\right)$, and introduce the perturbation variables $u, v, w, z, y$ and $s$ which are defined by

$$
\mathcal{I}=\mathcal{I}_{\mathrm{e}}+u, \quad \mathcal{D}=\mathcal{D}_{\mathrm{e}}+v, \quad \mathcal{C}=\mathcal{C}_{\mathrm{e}}+w, \quad \mathcal{L}=\mathcal{L}_{\mathrm{e}}+z, \quad \mathcal{L}_{\mathrm{ox}}=\mathcal{L}_{\text {oxe }}+y \text { and } \mathcal{R}=\mathcal{R}_{\mathrm{e}}+s .
$$

Substituting the assumed form for $\mathcal{I}-\mathcal{R}$ into (1)-(6) and keeping only terms that are linear in the perturbation variables results in the system of equations

$$
\begin{gathered}
\frac{\partial u}{\partial t}=\operatorname{div}\left(\mu_{1} \nabla u\right)-\operatorname{div}(\chi \nabla w)-A u-B u-C u-D v-E y, \\
\frac{\partial v}{\partial t}=\operatorname{div}\left(\mu_{2} \nabla v\right)+F u-G u-H v-I v+J y, \\
\frac{\partial w}{\partial t}=\operatorname{div}\left(\mu_{3} \nabla w\right)-K u+L v-M w-N w \\
\frac{\partial z}{\partial t}=\operatorname{div}\left(\mu_{4} \nabla z\right)-P_{1} z+P_{2} y-P_{3} s \\
\frac{\partial y}{\partial t}=\operatorname{div}\left(\mu_{5} \nabla y\right)-Q_{1} u+Q_{2} z-Q_{3} y-Q_{4} y+Q_{5} s, \\
\frac{\partial s}{\partial t}=\operatorname{div}\left(\mu_{6} \nabla s\right)-R_{1} z-R_{2} s-R_{3} s
\end{gathered}
$$

with the boundary conditions

$$
\frac{\partial u}{\partial \mathbf{n}}=\frac{\partial v}{\partial \mathbf{n}}=\frac{\partial w}{\partial \mathbf{n}}=\frac{\partial z}{\partial \mathbf{n}}=\frac{\partial y}{\partial \mathbf{n}}=\frac{\partial s}{\partial \mathbf{n}}=0 .
$$

For ease of notation, we have introduced a number of parameters. The new parameters are defined to be:

$$
\begin{gathered}
A=d_{11}, \quad B=a_{15} \mathcal{L}_{\text {oxe }}, \quad C=a_{12} \mathcal{D}_{\mathrm{e}}, \quad D=a_{12} \mathcal{I}_{\mathrm{e}}, \quad E=a_{15} \mathcal{I}_{\mathrm{e}}, \\
F=c_{15} \mathcal{L}_{\text {oxe }}, \quad G=a_{21} \mathcal{D}_{\mathrm{e}}, \quad H=a_{21} \mathcal{I}_{\mathrm{e}}, \quad I=d_{22}, \quad J=c_{15} \mathcal{I}_{\mathrm{e}}, \\
K=a_{31} \mathcal{C}_{\mathrm{e}}, \quad L=p_{32}, \quad M=a_{31} \mathcal{I}_{\mathrm{e}}, \quad N=d_{33}, \quad P_{1}=a_{46} \mathcal{R}_{\mathrm{e}}, \quad P_{2}=b_{4} A_{\mathrm{ox}} r_{4}, \\
P_{3}=a_{46} \mathcal{L}_{\mathrm{e}}, \quad Q_{1}=b_{15} \mathcal{L}_{\text {oxe }}, \quad Q_{2}=c_{46} \mathcal{R}_{\mathrm{e}}, \quad Q_{3}=A_{\mathrm{ox}} r_{4}, \quad Q_{4}=b_{15} \mathcal{I}_{\mathrm{e}}, \\
Q_{5}=c_{46} \mathcal{L}_{\mathrm{e}}, \quad R_{1}=b_{46} \mathcal{R}_{\mathrm{e}}, \quad R_{2}=b_{46} \mathcal{L}_{\mathrm{e}}, \quad R_{3}=b_{6} A_{\mathrm{ox}} \quad \text { and } \chi=\chi\left(\mathcal{I}_{\mathrm{e}}, \mathcal{C}_{\mathrm{e}}\right) .
\end{gathered}
$$

Each of these constants is assumed to be non-negative. Note that due to balance of mass $F=B+Q_{1}, J=E+Q_{4}, Q_{2}=P_{1}+R_{1}$ and $Q_{5}=P_{3}+R_{2}$. In our analysis, we will 
make the simplifying assumption that the mobility and diffusions coefficient $\mu_{i}$, $i=1, \ldots, 6$ are constant.

Let $\vec{U}=(u, v, w, z, y, s)$. Before proceeding, we define stability in the following way:

Definition. The equilibrium state is called asymptotically stable if every solution of the linearized initial boundary value problem (7)-(13) for the perturbation variables vanishes at infinity in the sense that there exists a positive functional

$$
\mathcal{F}(\vec{U})=\Phi(t) \quad \text { such that } \quad \lim _{t \rightarrow \infty} \Phi(t)=0 .
$$

We will adapt the method used in [9], to build appropriate functionals for two cases of the system (7)-(13) before turning attention to a system with non-homogeneous boundary conditions. In the first of these cases, we assume that the integrals of the products $u v$ and $u w$ over $\Omega$ are positive. Physically, this can be interpreted as saying that an increase debris $(v>0)$ and an increase in chemoattractant $(w>0)$ results in an increase in immune cells $(u>0)$. Likewise a decrease in debris and chemoattractant $(v<0, w<0)$ is met with a decrease in immune cells $(u<0)$. This is physically reasonable. However, we will show that this condition can be dropped and a slightly weaker stability condition can be obtained.

\subsection{Case A: $\int_{\Omega} \mathrm{uv} \mathrm{dx}>0$ and $\int_{\boldsymbol{\Omega}} \mathrm{uw} \mathrm{dx}>0$}

In this section, we introduce several integrals. For ease of notation, we will suppress the integration variables. All integration is over the domain $\Omega$ unless otherwise specifically indicated.

The transition matrix characterizing the species interactions associated with the system (7)-(12) is

$$
\Lambda=\left[\begin{array}{cccccc}
-(A+B+C) & -D & 0 & 0 & -E & 0 \\
F-G & -(H+I) & 0 & 0 & J & 0 \\
-K & L & -(M+N) & 0 & 0 & 0 \\
0 & 0 & 0 & -P_{1} & P_{2} & -P_{3} \\
Q_{1} & 0 & 0 & Q_{2} & -\left(Q_{3}+Q_{4}\right) & Q_{5} \\
0 & 0 & 0 & -R_{1} & 0 & -\left(R_{2}+R_{3}\right)
\end{array}\right] .
$$

We will assume that the eigenvalues of $\Lambda$ have negative real part. ${ }^{6}$ In the following construction, this ensures that $\int U_{i} \rightarrow 0$ as $t \rightarrow \infty$ for $U_{i}=u, v, w, z, y$ or $s$. This follows from Green's theorem and the homogeneous Neumann boundary conditions. This constraint does not guarantee stability of the system or even point-wise boundedness of each $U_{i}$. We will also assume here that $\mu_{2}=0$ which is consistent with the immobile nature of the lesion core.

Throughout the construction of an appropriate functional, we will make judicious use of the inequalities

$$
\begin{aligned}
\text { (Cauchy) } a b & \leq \varepsilon a^{2}+\frac{1}{4 \varepsilon} b^{2}, \quad \text { and } \\
\text { (Poincaré) } \int_{\Omega} u^{2} & \leq \frac{1}{|\Omega|}\left(\int_{\Omega} u\right)^{2}+C_{\mathrm{p}} \int_{\Omega}|\nabla u|^{2} .
\end{aligned}
$$


The parameter $C_{\mathrm{p}}$ present in the Poincare inequality is a constant that depends on the geometry of the domain. When an $L^{2}$ norm is considered, $C_{\mathrm{p}}$ is related to the inverse of the first positive eigenfrequency of a free membrane [1]. With the constraint that the eigenvalues of $\Lambda$ have negative real part, each of the integrals $\left(\int U_{i}\right)^{2}$ will decay exponentially. So, for simplicity, we will ignore these terms from the beginning of our construction.

We begin by multiplying (7) by $u$, (8) by $v$ and so forth. Integration by parts and application of the Poincaré and Cauchy inequalities to several terms yields the preliminary inequalities:

$$
\begin{gathered}
\frac{1}{2} \partial_{t} \int u^{2} \leq \int\left[-\left(A_{1}+\frac{C_{\mathrm{p}}}{2}\left(\mu_{1}-\chi / 2\right)-\frac{D+E}{2}\right) u^{2}+\frac{D}{2} v^{2}+\frac{E}{2} y^{2}-\frac{\mu_{1}}{2}|\nabla u|^{2}+\frac{\chi}{2}|\nabla w|^{2}\right] \\
\frac{1}{2} \partial_{t} \int v^{2} \leq \int\left[-G_{1} u v-\left(H_{1}-\frac{J}{2}\right) v^{2}+\frac{J}{2} y^{2}\right] \\
\frac{1}{2} \partial_{t} \int w^{2} \leq \int\left[-K u w+\frac{L}{2} v^{2}-\left(M_{1}+\frac{C_{\mathrm{p}}}{2} \mu_{3}-\frac{L}{2}\right) w^{2}-\frac{\mu_{3}}{2}|\nabla w|^{2}\right] \\
\frac{1}{2} \partial_{t} \int z^{2} \leq \int\left[-\left(P_{1}+C_{\mathrm{p}} \mu_{4}-\frac{P_{2}+P_{3}}{2}\right) z^{2}+\frac{P_{2}}{2} y^{2}+\frac{P_{3}}{2} s^{2}\right] \\
\frac{1}{2} \partial_{t} \int y^{2} \leq \int\left[\frac{Q_{1}}{2} u^{2}+\frac{Q_{2}}{2} z^{2}-\left(Q_{3}+Q_{4}+C_{\mathrm{p}} \mu_{5}-\frac{Q_{1}+Q_{2}+Q_{5}}{2}\right) y^{2}+\frac{Q_{5}}{2} s^{2}\right] \\
\frac{1}{2} \partial_{t} \int s^{2} \leq \int\left[\frac{R_{1}}{2} z^{2}-\left(R_{2}+R_{3}+C_{\mathrm{p}} \mu_{6}-\frac{R_{1}}{2}\right) s^{2}\right]
\end{gathered}
$$

For ease of notation, we set

$$
A_{1}=A+B+C, G_{1}=G-F, H_{1}=H+I \text { and } M_{1}=M+N .
$$

To proceed, we multiply (7) by $u_{t} / D$ and use the equality from (9)

$$
\nabla^{2} w=\frac{1}{\mu_{3}}\left(w_{t}+K u-L v+M_{1} w\right)
$$

to arrive at

$$
\begin{aligned}
\frac{1}{D} \int\left(u_{t}\right)^{2} \leq & \int\left[-\frac{\mu_{1}}{2 D} \partial_{t}|\nabla u|^{2}-\frac{\chi}{\mu_{3} D} u_{t} w_{t}-\left(\frac{\chi K}{2 \mu_{3} D}+\frac{A_{1}}{2 D}\right) \partial_{t} u^{2}\right. \\
& \left.+\frac{\chi L}{2 \mu_{3} D}\left(u_{t}\right)^{2}+\frac{\chi L}{2 \mu_{3} D} v^{2}-\frac{\chi M_{1}}{\mu_{3} D} u_{t} w-u_{t} v+\frac{E}{2 D}\left(u_{t}\right)^{2}+\frac{E}{2 D} y^{2}\right]
\end{aligned}
$$

We can further separate the second term on the right-hand side by imposing the Cauchy inequality. That is

$$
\frac{\chi}{\mu_{3} D} u_{t} w_{t} \leq \frac{\chi}{4 \varepsilon \mu_{3} D}\left(u_{t}\right)^{2}+\frac{\varepsilon \chi}{\mu_{3} D}\left(w_{t}\right)^{2} .
$$


Later, $\varepsilon$ will be specified as needed. We impose the conditions:

[Condition 1] $E<1$,

$$
\begin{gathered}
\text { [Condition 2] } \frac{\chi L}{2 \mu_{3}}<\frac{1}{4} \text { and } \\
\text { [Condition3] } \frac{\chi}{4 \varepsilon \mu_{3}}<\frac{1}{8} .
\end{gathered}
$$

This will allow us to move all terms involving $\left(u_{t}\right)^{2}$ to the left. We have

$$
\begin{aligned}
\frac{1}{8 D} \int\left(u_{t}\right)^{2} \leq & \int\left[-\frac{\mu_{1}}{2 D} \partial_{t}|\nabla u|^{2}+\frac{\varepsilon \chi}{\mu_{3} D}\left(w_{t}\right)^{2}-\left(\frac{\chi K}{2 \mu_{3} D}+\frac{A_{1}}{2 D}\right) \partial_{t} u^{2}\right. \\
& \left.+\frac{\chi L}{2 \mu_{3} D} v^{2}-\frac{\chi M_{1}}{\mu_{3} D} u_{t} w-u_{t} v+\frac{E}{2 D} y^{2}\right] .
\end{aligned}
$$

Next, we want to use Equation (9) to investigate the term $\left(\varepsilon \chi / \mu_{3} D\right)\left(w_{t}\right)^{2}$ appearing in (22), and choose an advantageous value for $\varepsilon$. If we multiply (9) by $2 w_{t}$, integrate by parts and use the Cauchy inequality on the product $v w_{t}$, we have

$$
\int 2\left(w_{t}\right)^{2} \leq \int\left[-\mu_{3} \partial_{t}|\nabla w|^{2}-2 K u w_{t}+L v^{2}+L\left(w_{t}\right)^{2}-M_{1} \partial_{t} w^{2}\right] .
$$

Imposing

$$
\text { [Condition 4] } L<1 \text {, }
$$

$$
\frac{\varepsilon \chi}{\mu_{3} D} \int\left(w_{t}\right)^{2} \leq \int\left[-\frac{\varepsilon \chi}{D} \partial_{t}|\nabla w|^{2}-\frac{2 \varepsilon \chi K}{\mu_{3} D} u w_{t}+\frac{\varepsilon \chi L}{\mu_{3} D} v^{2}-\frac{\varepsilon \chi M_{1}}{\mu_{3} D} \partial_{t} w^{2}\right] \text {. }
$$

Finally, we can substitute this into Equation (22). If in addition, we set

$$
\varepsilon=\frac{M_{1}}{2 K}
$$

we can collect the products $u_{t} w$ and $u w_{t}$ into a single term. The resulting inequality is

$$
\begin{aligned}
\frac{1}{8 D} \int\left(u_{t}\right)^{2} \leq & \int\left[-\frac{\mu_{1}}{2 D} \partial_{t}|\nabla u|^{2}-\frac{\chi M_{1}}{2 K D} \partial_{t}|\nabla w|^{2}-\frac{\chi M_{1}}{\mu_{3} D} \partial_{t}(u w)-u_{t} v\right. \\
& \left.-\left(\frac{\chi K}{2 \mu_{3} D}+\frac{A_{1}}{2 D}\right) \partial_{t} u^{2}-\frac{\chi M_{1}^{2}}{2 K \mu_{3} D} \partial_{t}(w)^{2}+\left(\frac{\chi L}{2 \mu_{3} D}+\frac{\chi M_{1} L}{2 K \mu_{3} D}\right) v^{2}+\frac{E}{2 D} y^{2}\right] .
\end{aligned}
$$

Inequality (23) is one of the principal inequalities to be used in the current construction. Here, we simply note that by our definition of $\varepsilon$ the previous [Condition 3] can be restated as

$$
\frac{\chi K}{2 M_{1} \mu_{3}}<\frac{1}{8}
$$

To continue, we assume

$$
\text { [Condition 5] } G_{1}=G-F>0 \text { and }
$$

[Condition 6] $J<1$. 
Then, if we multiply both sides of $(8)$ by $\left(1 / G_{1}\right) v_{t}$ and integrate, we can obtain

$$
\frac{1}{2 G_{1}} \int\left(v_{t}\right)^{2} \leq \int\left[-u v_{t}-\frac{H_{1}}{2 G_{1}} \partial_{t} v^{2}+\frac{J}{2 G_{1}} y^{2}\right] .
$$

Now, we can combine (14)-(19), (23) and (24) to obtain the first major inequality. In so doing, we will move all terms involving a time derivative to the left and ignore terms of the form $\left(u_{t}\right)^{2},\left(v_{t}\right)^{2}$ and $\left(w_{t}\right)^{2}$.

$$
\begin{aligned}
& \int \partial_{t}\left[\left(\frac{1}{2}+\frac{\chi K}{2 \mu_{3} D}+\frac{A_{1}}{2 C}\right) u^{2}+\left(\frac{1}{2}+\frac{H_{1}}{2 G_{1}}\right) v^{2}+\left(\frac{1}{2}+\frac{\chi M_{1}^{2}}{2 K \mu_{3} D}\right) w^{2}\right. \\
& \left.+\frac{1}{2} z^{2}+\frac{1}{2} y^{2}+\frac{1}{2} s^{2}+(u v)+\frac{\chi M_{1}}{\mu_{3} D}(u w)+\frac{\mu_{1}}{2 D}|\nabla u|^{2}+\frac{\chi M_{1}}{2 K D}|\nabla w|^{2}\right] \leq \\
& -\int\left[C_{u} u^{2}+C_{v} v^{2}+C_{w} w^{2}+C_{z} z^{2}+C_{y} y^{2}+C_{s} s^{2}+C_{u v}(u v)\right. \\
& \left.+C_{u w}(u w)+C_{\nabla u}|\nabla u|^{2}+C_{\nabla w}|\nabla w|^{2}\right] .
\end{aligned}
$$

The coefficients on the right-hand side are

$$
\begin{aligned}
C_{u} & =A_{1}+\frac{C_{\mathrm{p}}}{2}\left(\mu_{1}-\frac{\chi}{2}\right)-\frac{D+E+Q_{1}}{2}, \\
C_{v} & =H_{1}-\frac{D+J+L}{2}-\frac{\chi L}{2 \mu_{3} D}-\frac{\chi M_{1} L}{2 K \mu_{3} D}, \\
C_{w} & =M_{1}+\frac{C_{\mathrm{p}}}{2}\left(\mu_{3}-\frac{\chi}{2}\right)-\frac{L}{2}, \\
C_{z} & =P_{1}+\mu_{4} C_{\mathrm{p}}-\frac{P_{2}+P_{3}+Q_{2}+R_{1}}{2}, \\
C_{y} & =Q_{3}+Q_{4}+\mu_{5} C_{\mathrm{p}}-\frac{P_{2}+Q_{1}+Q_{2}+Q_{5}+E+J}{2}-\frac{E}{2 D}-\frac{J}{2 G_{1}}, \\
C_{s} & =R_{2}+R_{3}+\mu_{6} C_{\mathrm{p}}-\frac{P_{3}+Q_{5}+R_{1}}{2}, \\
C_{u v} & =G_{1}, \\
C_{u w} & =K, \\
C_{\nabla u} & =\frac{1}{2}\left(\mu_{1}-\frac{\chi}{2}\right) \\
C_{\nabla w} & =\frac{1}{2}\left(\mu_{3}-\frac{\chi}{2}\right) .
\end{aligned}
$$

We are ready to state our first major result.

TheOREM 1. The equilibrium solution $\left(\mathcal{I}_{\mathrm{e}}, \mathcal{D}_{\mathrm{e}}, \mathcal{C}_{\mathrm{e}}, \mathcal{L}_{\mathrm{e}}, \mathcal{L}_{\text {oxe }}, \mathcal{R}_{\mathrm{e}}\right)$ of (1)-(6) subject to the homogeneous Neumann boundary conditions is asymptotically stable provided

(i) $\int u v>0$ and $\int u w>0$

(ii) all eigenvalues of $\Lambda$ have negative real part,

(iii) Conditions 1-6 hold, and

(iv) $M=\min \left(C_{u}, C_{v}, C_{w}, C_{z}, C_{y}, C_{s}, C_{u v}, C_{u w}, C_{\nabla u}, C_{\nabla w}\right)>0$ 
The proof requires a definition of the functional as the obvious modification of the lefthand side of (25). Of interest is the physical interpretations of the sufficiency conditions stated here. The first has already been discussed.

As for the eigenvalues of $\Lambda$, if $E \ll 1$ and $J \ll 1$ (Conditions 1 and 6) and $Q_{1} \ll 1$, then to leading order, the matrix is block diagonal. Each of these parameters being small indicates weak foam cell production since $E, J$ and $Q_{1}$ are rates at which immune cells and oxLDL are transformed into debris. Then, if $Q_{2} \ll 1$ and $R_{1} \ll 1$, the lower block would have eigenvalues $-P_{1},-\left(Q_{3}+Q_{4}\right)$ and $-\left(R_{2}+R_{3}\right)$ which are all negative. Now, $Q_{2}$ and $R_{1}$ are rates of oxidation of LDL, a destabilizing reaction, whereas $\left(Q_{3}+Q_{4}\right)$ and $\left(R_{2}+R_{3}\right)$ are rates of healthy restoration due to anti-oxidant reaction. So, these eigenvalues being negative indicates dominance of anti-oxidant reactions over oxidation of LDL. This is physically realistic as a stability - i.e. indication of healthiness condition. As for the upper block under the conditions $E \ll 1$ and $J \ll 1$ and $Q_{1} \ll 1$, if $L \ll 1$ (Condition 4) so that production of chemoattractant is small, then to leading order the eigenvalues of the upper block are

$$
-M_{1}, \quad-\frac{1}{2}\left(H_{1}+A_{1}\right) \pm \sqrt{\left(H_{1}+A_{1}\right)^{2}-4\left(H_{1} A_{1}-D G\right)} .
$$

Large $M_{1}$ indicates low levels of the chemoattractant consistent with low inflammation, while large $A_{1}$ (due to $A$ and $C$ ) and $H_{1}$ indicate healthy immune function since these are rates of decrease of immune cells and debris due to normal immune response. Large $D$ and $G_{1}$ also indicate healthy immune response. The eigenvalues have negative real part provided $\sqrt{\left.\left(H_{1}-A_{1}\right)^{2}+4 D G\right)}<H_{1}+A_{1}$, which is also physically reasonable in the sense that this would be associated with stabilizing effects dominating.

Next, the meaning of Conditions 1, 4 and 6 have been given. Conditions 2 and 3 hold if the diffusion due to $\mu_{3}$ dominates the chemotactic effects due to $\chi$. This is well known as stabilizing in any system characterized by chemotaxis. Finally, Condition 5 holds if removal of debris due to normal immune function $(G)$ is large compared to foam cell production due to binding of macrophages to oxLDL $(F)$. This is a physically relevant condition.

Finally, note that each of the coefficients on the right-hand side in (25) have positive and negative part (in order from left to right). The last item of the theorem holds if diffusion dominates chemotactic effects $\left(C_{u}, C_{w}, C_{z}, C_{y}, C_{s}, C_{\nabla u}, C_{\nabla_{w}}>0\right)$, healthy immune response dominates inflammation $\left(A_{1}, G_{1}, H_{1}, K\right.$ and $M_{1}$ are large, $L$ small), and anti-oxidant effects dominate over oxidation $\left(Q_{2}, R_{1}\right.$ and $Q_{5}$ are small compared to $Q_{3}+Q_{4}$ and $R_{2}+R_{3}$ ). Note that each of these is naturally consistent with stability.

We note here that (25) can be rewritten in the form

$$
\frac{\mathrm{d}}{\mathrm{d} t} \int \vec{\xi} \cdot \mathbf{A}_{1} \vec{\xi} \leq-\int \vec{\xi} \cdot \mathbf{A}_{2} \vec{\xi}
$$

where $\vec{\xi}=(u, v, w, z, y, s,|\nabla u|,|\nabla w|)^{\mathrm{T}}$

$$
\mathbf{A}_{1}=\left[\begin{array}{ccccc}
d_{1} & \frac{1}{2} & \frac{\chi M_{1}}{2 \mu_{3} D} & \cdots & 0 \\
\frac{1}{2} & d_{2} & \cdots & \cdots & 0 \\
\frac{\chi M_{1}}{2 \mu_{3} D} & 0 & d_{3} & \cdots & 0 \\
0 & 0 & 0 & d_{4} & \cdots \\
\vdots & \vdots & \vdots & \vdots & \vdots
\end{array}\right]
$$


and

$$
\mathbf{A}_{2}=\left[\begin{array}{ccccc}
C_{u} & \frac{C_{u v}}{2} & \frac{C_{u v}}{2} & \cdots & 0 \\
\frac{C_{u v}}{2} & C_{v} & 0 & \cdots & 0 \\
\frac{C_{u w}}{2} & 0 & C_{w} & \cdots & 0 \\
\vdots & \vdots & \vdots & \vdots & \vdots
\end{array}\right] .
$$

For asymptotic stability, it is sufficient that $\mathbf{A}_{1}$ and $\mathbf{A}_{2}$ be positive definite (See the Lemma in Appendix A). This general result can be verified for any particular set of the parameter values if such data becomes available. Unfortunately, in contrast to Theorem 1, this is difficult to evaluate from a bio-physical perspective in the absence of numerical data. Theorem 1, however, gives an explicit set of inequalities involving the pivotal parameters of the system. To overcome the discrepancy for the case when the integrals $\int u v$ and $\int u w$ can change signs, we use the modified construction that follows.

\subsection{Case B: Dropping the assumptions $\int u v, \int u w>0$}

The preceding theorem requires $\int u v, \int u w>0$. We can drop this requirement and obtain a slightly different result. We require a regrouping of the terms in our inequality (25) to eliminate the products $u v$ and $u w$ in favour of terms of the form $(u+v)^{2}$ and $(\alpha u+\beta w)^{2}$ for some constants $\alpha$ and $\beta$. To this end, first note that

$$
\frac{1}{2} u^{2}+u v+\frac{1}{2} v^{2}=\frac{1}{2}(u+v)^{2} .
$$

A regrouping as this leaves us with

$$
\left(\frac{\chi K}{2 \mu_{3} D}+\frac{A_{1}}{2 D}\right) u^{2}+\left(\frac{1}{2}+\frac{\chi M_{1}^{2}}{2 K \mu_{3} D}\right) w^{2}+\frac{\chi M_{1}}{\mu_{3} D}(u w),
$$

still appearing on the left in (25). We can restate these terms as

$$
\Phi u^{2}+\Psi w^{2}+(\alpha u+\beta w)^{2}
$$

by setting

$$
\alpha=\sqrt{\frac{\chi K}{2 \mu_{3} D}}, \quad \beta=M_{1} \sqrt{\frac{\chi}{2 \mu_{3} D}}, \quad \Phi=\frac{A_{1}}{2 D} \text { and } \Psi=\frac{1}{2} .
$$

Let the vector $\mathbf{Y}$ be defined component wise by

$$
\begin{gathered}
Y_{1}=\int \frac{A_{1}}{2 D} u^{2}, \quad Y_{2}=\int \frac{H_{1}}{2 G_{1}} v^{2}, \quad Y_{3}=\int \frac{1}{2} w^{2}, \quad Y_{4}=\int \frac{1}{2} z^{2}, \\
Y_{5}=\int \frac{1}{2} y^{2}, \quad Y_{6}=\int \frac{1}{2} s^{2}, \quad Y_{7}=\int \frac{1}{2}(u+v)^{2}, \\
Y_{8}=\int(\alpha u+\beta w)^{2}, \quad Y_{9}=\int \frac{\mu_{1}}{2 D}|\nabla u|^{2}, \quad Y_{10}=\int \frac{\chi M_{1}}{2 K D}|\nabla w|^{2},
\end{gathered}
$$


and consider the functional $\mathcal{F}_{1}(\mathbf{Y})=\sum Y_{i}$. Obviously, $\mathcal{F}_{1}(\mathbf{Y})$ is non-negative and vanishes only if $\mathbf{Y}=0$.

Next, we seek a reordering of the relevant terms on the right-hand side of (25). However, for the case under consideration, let us replace (25) with a similar inequality obtained by replacing (14) with the following:

$$
\frac{1}{2} \partial_{t} \int u^{2} \leq \int\left[-\left(A_{1}-\frac{E}{2}\right) u^{2}+\frac{E}{2} y^{2}-D u v-\left(\mu_{1}-\frac{\chi}{2}\right)|\nabla u|^{2}+\frac{\chi}{2}|\nabla w|^{2}\right] .
$$

Note that this differs from (14) only by the term - Duv; here, we withhold applying the Cauchy inequality to the product $D u v$, while all other terms are unchanged. We obtain the inequality (25) with the modification that the coefficients $C_{u}, C_{v}$ and $C_{u v}$ replaced by $\tilde{C}_{u}, \tilde{C}_{v}$ and $\tilde{C}_{u v}$ defined by

$$
\begin{gathered}
\tilde{C}_{u}=A_{1}+\frac{C_{\mathrm{p}}}{2}\left(\mu_{1}-\frac{\chi}{2}\right)-\frac{E+Q_{1}}{2}, \\
\tilde{C}_{v}=H_{1}-\frac{J+L}{2}-\frac{\chi L}{2 \mu_{3} D}-\frac{\chi M_{1} L}{2 K \mu_{3} D} \\
\tilde{C}_{u v}=G_{1}+D .
\end{gathered}
$$

Set

$$
\alpha_{u}=\sqrt{\frac{1}{2} \tilde{C}_{u}}, \quad \alpha_{v}=\sqrt{\tilde{C}_{v}} \text { and } \alpha_{w}=\sqrt{C_{w}} .
$$

Then, under the conditions

$$
\text { [Condition 7] } \alpha_{u} \alpha_{v} \geq G_{1}+D, \quad \text { and }
$$

$$
\text { [Condition 8] } \alpha_{u} \alpha_{w} \geq K
$$

the inequalities

$$
\begin{aligned}
-\left(\frac{1}{2} \tilde{C}_{u} u^{2}+\tilde{C}_{u v} u v+\tilde{C}_{v} v^{2}\right) & \leq-\frac{1}{2}\left(\alpha_{u} u+\alpha_{v} v\right)^{2} \\
-\left(\frac{1}{2} \tilde{C}_{u} u^{2}+C_{u w} u w+C_{w} w^{2}\right) & \leq-\frac{1}{2}\left(\alpha_{u} u+\alpha_{w} w\right)^{2},
\end{aligned}
$$

hold.

Now, we define the functional $\mathcal{F}_{2}$ as

$$
\begin{aligned}
& \mathcal{F}_{2}\left(u, v, w, z, y, s,\left(\alpha_{u} u+\alpha_{v} v\right),\left(\alpha_{u} u+\alpha_{w} w\right),|\nabla u|,|\nabla w|\right) \\
& \quad=\int\left[\tilde{C}_{z} z^{2}+\tilde{C}_{y} y^{2}+\tilde{C}_{s} s^{2}+\frac{1}{2}\left(\alpha_{u} u+\alpha_{v} v\right)^{2}+\frac{1}{2}\left(\alpha_{u} u+\alpha_{w} w\right)^{2}+\tilde{C}_{\nabla u}|\nabla u|^{2}+\tilde{C}_{\nabla w}|\nabla w|^{2}\right] .
\end{aligned}
$$


The coefficients are related to the previous ones by

$$
\begin{aligned}
\tilde{C}_{z} & =\frac{C_{z}}{1 / 2}, \\
\tilde{C}_{y} & =\frac{C_{y}}{1 / 2} \\
\tilde{C}_{s} & =\frac{C_{s}}{1 / 2} \\
\tilde{C}_{\nabla u} & =\frac{C_{\nabla u}}{\mu_{1} /(2 D)}, \\
\tilde{C}_{\nabla w} & =\frac{C_{\nabla u}}{\left(\chi M_{1}\right) /(2 K D)} .
\end{aligned}
$$

Let $M=\min \left(\tilde{C}_{z}, \ldots, \tilde{C}_{\nabla w}\right)$. Then, note that

$$
\frac{\mathrm{d}}{\mathrm{d} t} \mathcal{F}_{1}(\mathbf{Y}) \leq-M \mathcal{F}_{2}\left(0,0,0, Y_{4}, Y_{5}, Y_{6}, Y_{7}, Y_{8}, Y_{9}, Y_{10}\right)
$$

Hence, the perturbations $u, \ldots, s$ decay, and the equilibrium solution

$$
\left(\mathcal{I}_{\mathrm{e}}, \mathcal{D}_{\mathrm{e}}, \mathcal{C}_{\mathrm{e}}, \mathcal{L}_{\mathrm{e}}, \mathcal{L}_{\text {oxe }}, \mathcal{R}_{\mathrm{e}}\right)
$$

is asymptotically stable ${ }^{7}$ provided the conditions of Theorem 1 as well as the additional Conditions 7 and 8 hold.

\section{Non-homogeneous boundary conditions}

Here, we return our attention to the original system (1)-(6) considered with the new boundary conditions imposed:

$$
\begin{gathered}
\frac{\partial \mathcal{D}}{\partial \mathbf{n}}=\frac{\partial \mathcal{L}_{\mathrm{ox}}}{\partial \mathbf{n}}=\frac{\partial \mathcal{R}}{\partial \mathbf{n}}=0 \\
\mu_{1} \frac{\partial \mathcal{I}}{\partial \mathbf{n}}=\alpha_{1}\left(\mathcal{C}-\mathcal{C}_{*}\right), \quad \mu_{3} \frac{\partial \mathcal{C}}{\partial \mathbf{n}}=-\alpha_{3}\left(\mathcal{C}-\mathcal{C}_{*}\right) \text { and } \mu_{4} \frac{\partial \mathcal{L}}{\partial \mathbf{n}}=-\alpha_{4}\left(\mathcal{L}-\mathcal{L}_{B}\right),
\end{gathered}
$$

on $\Gamma_{1}$. The parameters $\alpha_{1}$ and $\alpha_{3}$ are positive constants as is $\mathcal{C}_{*}$. The parameter $\mathcal{C}_{*}$ represents baseline level of chemoattractant in the blood stream. If the level of chemoattractant at the endothelial layer is greater than the baseline level, chemoattractant enters the blood stream while immune cells enter into the subendothelial intima. The parameter $\mathcal{L}_{B}$ is the serum level of LDL. Both forward and reverse transport of LDL from the plasma and intimal layer can be considered depending on the sign of the coefficient $\alpha_{4}$. It is well documented that high serum LDL levels are positively associated with arterial lesions. Allowing the transport of LDL will allow us to arrive at a stability criteria that relates this level to other significant parameters. We still consider homogeneous boundary conditions for all species on the outer boundary $\Gamma_{2}$.

Allowing for transport across the boundary in more realistic, yet presents us with additional mathematical complexity. The primary problem arises when we integrate by parts as non-zero boundary integrals must be considered. In the following construction, we make appropriate modifications.

We again linearize the system (1)-(6) about the constant equilibrium state $\left(\mathcal{I}_{\mathrm{e}}, \mathcal{D}_{\mathrm{e}}, \mathcal{C}_{\mathrm{e}}, \mathcal{L}_{\mathrm{e}}, \mathcal{L}_{\text {oxe }}, \mathcal{R}_{\mathrm{e}}\right)$. It is necessary here that $\mathcal{C}_{\mathrm{e}}=\mathcal{C}_{*}$ and $\mathcal{L}_{\mathrm{e}}=\mathcal{L}_{B}$. Let us denote 
the perturbation variables $u, v, w, z, y$ and $s$ which are defined as before by

$$
\begin{gathered}
\mathcal{I}=\mathcal{I}_{\mathrm{e}}+u, \quad \mathcal{D}=\mathcal{D}_{\mathrm{e}}+v, \quad \mathcal{C}=\mathcal{C}_{\mathrm{e}}+w, \quad \mathcal{L}=\mathcal{L}_{\mathrm{e}}+z, \\
\mathcal{L}_{\mathrm{ox}}=\mathcal{L}_{\text {oxe }}+y \text { and } \mathcal{R}=\mathcal{R}_{\mathrm{e}}+s .
\end{gathered}
$$

Upon linearization, we find that $u, v, w, z, y$ and $s$ satisfy Equations (7)-(12) as before. The boundary conditions for the system now under consideration are unchanged for $v, y$ and $s$. That is

$$
\frac{\partial v}{\partial \mathbf{n}}=\frac{\partial y}{\partial \mathbf{n}}=\frac{\partial s}{\partial \mathbf{n}}=0
$$

For $u, w$ and $z$ we note that

$$
\mu_{1} \frac{\partial\left(\mathcal{I}_{\mathrm{e}}+u\right)}{\partial \mathbf{n}}=\alpha_{1}\left(\mathcal{C}_{\mathrm{e}}+w-\mathcal{C}_{*}\right) \quad \text { so } \quad \mu_{1} \frac{\partial u}{\partial \mathbf{n}}=\alpha_{1} w
$$

Similarly

$$
\mu_{3} \frac{\partial w}{\partial \mathbf{n}}=-\alpha_{3} w \text { and } \mu_{4} \frac{d z}{d \mathbf{n}}=-\alpha_{4} z \quad \text { on } \Gamma_{1} .
$$

We will construct an inequality that allows us to conclude sufficient conditions under which the equilibrium state is stable. To address the impact of the boundary conditions, we will use the following inequalities:

$$
\begin{gathered}
\text { (Sobolev) } \int_{\Gamma} u^{2} \mathrm{~d} s \leq C_{1}\left(\int_{\Omega} u^{2}+|\nabla u|^{2}\right) \mathrm{d} x \text { and } \\
\text { (Generalized Friedrich) } \quad C_{2} \int_{\Omega} u^{2} \mathrm{~d} x \leq \int_{\Omega}|\nabla u|^{2} \mathrm{~d} x+C_{3} \int_{\Gamma} u^{2} \mathrm{~d} s .
\end{gathered}
$$

The coefficients $C_{1}, C_{2}$ and $C_{3}$ depend on the geometry ${ }^{8}$ of the domain $\Omega$ with boundary $\Gamma$.

We proceed in a fashion similar to the previous cases by multiplying (7) by $u$, (8) by $v$, and so forth and integrating by parts to obtain (all integration that follows is over $\Omega$ except where specifically indicated)

$$
\begin{gathered}
\frac{1}{2} \partial_{t} \int u^{2}=\int_{\Gamma_{1}} u w\left(\alpha_{1}+\frac{\chi \alpha_{3}}{\mu_{3}}\right)-\mu_{1} \int|\nabla u|^{2}+\chi \int \nabla u \cdot \nabla w-A_{1} \int u^{2}-D \int u v-E \int u y, \\
\frac{1}{2} \partial_{t} \int v^{2}=-G_{1} \int u v-H_{1} \int v^{2}+J \int v y \\
\frac{1}{2} \partial_{t} \int w^{2}=-\alpha_{3} \int_{\Gamma_{1}} w^{2}-\mu_{3} \int|\nabla w|^{2}-K \int u w+L \int v w-M_{1} \int w^{2} \\
\frac{1}{2} \partial_{t} \int z^{2}=-\alpha_{4} \int_{\Gamma_{1}} z^{2}-\mu_{4} \int|\nabla z|^{2}-P_{1} \int z^{2}+P_{2} \int y z-P_{3} \int z s \\
\frac{1}{2} \partial_{t} \int y^{2}=-\mu_{5} \int|\nabla y|^{2}-Q_{1} \int u y+Q_{2} \int z y-\left(Q_{3}+Q_{4}\right) \int y^{2}+Q_{5} \int y s \\
\frac{1}{2} \partial_{t} \int s^{2}=-\mu_{6} \int|\nabla s|^{2}-R_{1} \int z s-\left(R_{2}+R_{3}\right) \int s^{2} .
\end{gathered}
$$


We assume that the effect of foam cell formation on the concentration of oxLDL is negligible as compared to the competing oxidizing and anti-oxidant reactions. Thus, for simplicity, we set $Q_{1}=0$ and likewise $Q_{4}=0$. This is similar to our condition that $Q_{1}$ is small in the previous case considered. This of course requires that $c_{15}=a_{15}$.

Next, we can apply the Cauchy and Sobolev inequalities to (32) to arrive at the inequality

$$
\begin{aligned}
\frac{1}{2} \partial_{t} \int u^{2} \leq & \frac{1}{2} \int_{\Gamma_{1}} w^{2}\left(\alpha_{1}+\frac{\chi \alpha_{3}}{\mu_{3}}\right)-\left[\mu_{1}-C_{1}\left(\alpha_{1}+\frac{\chi \alpha_{3}}{\mu_{3}}\right)-\frac{\chi}{2}\right] \int|\nabla u|^{2} \\
& +\frac{\chi}{2} \int|\nabla w|^{2}-\left[A_{1}-\frac{C_{1}}{2}\left(\alpha_{1}+\frac{\chi \alpha_{3}}{\mu_{3}}\right)\right] \int u^{2}-D \int u v-E \int u y .
\end{aligned}
$$

Let us note that large $\mu_{3}$ should enhance the stability of the system in general, since this would indicate strong diffusive effects. Similarly, small $\alpha_{3}$ and small $\chi$ would be associated with stability since this corresponds to weak cumulative (in the domain and on the boundary) chemotactic effects. If $\mu_{3}$ is larger than $\chi \alpha_{3}$, we would expect this to be stabilizing. We impose the condition

$$
\text { [Condition 9] } \mu_{1}-C_{1}\left(\alpha_{1}+\frac{\chi \alpha_{3}}{\mu_{3}}\right)-\frac{\chi}{2} \equiv \bar{\mu}_{1} \geq 0 \text {. }
$$

This condition coupled with (38) implies

$$
\frac{1}{2} \partial_{t} \int u^{2} \leq \frac{1}{2} \int_{\Gamma_{1}} w^{2}\left(\alpha_{1}+\frac{\chi \alpha_{3}}{\mu_{3}}\right)-\left[A_{1}-\frac{C_{1}}{2}\left(\alpha_{1}+\frac{\chi \alpha_{3}}{\mu_{3}}\right)\right] \int u^{2}+\frac{\chi}{2} \int|\nabla w|^{2}-D \int u v-E \int u y .
$$

If we sum (34) and (39), we find that

$$
\begin{aligned}
\frac{1}{2} \partial_{t} \int\left(u^{2}+w^{2}\right) \leq & -\left[\alpha_{3}-\frac{1}{2}\left(\alpha_{1}+\frac{\chi \alpha_{3}}{\mu_{3}}\right)\right] \int_{\Gamma_{1}} w^{2}-\overline{\mu_{1}} \int|\nabla u|^{2}-\left(\mu_{3}-\frac{\chi}{2}\right) \int|\nabla w|^{2} \\
& -\left[A_{1}-\frac{C_{1}}{2}\left(\alpha_{1}+\frac{\chi \alpha_{3}}{\mu_{3}}\right)\right] \int u^{2}-D \int u v-E \int u y-K \int u w+L \int v w-M_{1} \int w^{2} .
\end{aligned}
$$

For ease of notation, we will introduce the parameter $\bar{\alpha}$

$$
\bar{\alpha}=\alpha_{3}\left[1-\frac{1}{2}\left(\frac{\alpha_{1}}{\alpha_{3}}+\frac{\chi}{\mu_{3}}\right)\right] .
$$

Similarly, we introduce the parameter $\bar{\mu}_{3}$ and impose the condition

$$
\text { [Condition 10] } \mu_{3}-\frac{\chi}{2} \equiv \overline{\mu_{3}} \geq 0 \text {. }
$$

Set $C\left(\bar{\alpha}, \bar{\mu}_{3}\right)=\min \left(\bar{\alpha} / C_{3}, \bar{\mu}_{3}\right)$. Then $C\left(\bar{\alpha}, \bar{\mu}_{3}\right)$ will increase if both $\bar{\alpha}$ and $\bar{\mu}_{3}$ increase, and is at least non-decreasing in $\bar{\alpha}$ and $\bar{\mu}_{3}$ independently. Letting $C=C_{2}$, where $C_{2}$ is the other geometrically dependent constant appearing in the generalized Friedrich inequality, we 
have (after applying said inequality)

$$
\bar{\alpha} \int_{\Gamma_{1}} w^{2}+\overline{\mu_{3}} \int_{\Omega}|\nabla w|^{2} \geq C\left(\bar{\alpha}, \overline{\mu_{3}}\right) C \int_{\Omega} w^{2} .
$$

One of the primary inequalities - that for sum $u^{2}+w^{2}-$ can now be written

$$
\begin{aligned}
\frac{1}{2} \frac{\mathrm{d}}{\mathrm{d} t} \int\left(u^{2}+w^{2}\right) \leq & -\left[A_{1}-\frac{C_{1}}{2}\left(\alpha_{1}+\frac{\chi \alpha_{3}}{\mu_{3}}\right)\right] \int u^{2}-\left[M_{1}+C\left(\bar{\alpha}, \overline{\mu_{3}}\right) C-\frac{L}{2}\right] \int w^{2} \\
& +\frac{L}{2} \int v^{2}-D \int u v-E \int u y-K \int u w .
\end{aligned}
$$

Additional inequalities are obtained from (33), (36) and (37), for $v^{2}, y^{2}$ and $s^{2}$, respectively.

$$
\begin{gathered}
\frac{1}{2} \frac{\mathrm{d}}{\mathrm{d} t} \int v^{2} \leq-G_{1} \int u v-\left(H_{1}-\frac{J}{2}\right) \int v^{2}+\frac{J}{2} \int y^{2} . \\
\frac{1}{2} \frac{\mathrm{d}}{\mathrm{d} t} \int y^{2} \leq-\left(\frac{\mu_{5}}{C_{\mathrm{p}}}+Q_{3}-\frac{Q_{2}+Q_{5}}{2}\right) \int y^{2}+\frac{Q_{2}}{2} \int z^{2}+\frac{Q_{5}}{2} \int s^{2}+\frac{\mu_{5}}{C_{\mathrm{p}}|\Omega|}\left(\int y\right)^{2} . \\
\frac{1}{2} \frac{\mathrm{d}}{\mathrm{d} t} \int s^{2} \leq-\left(\frac{\mu_{6}}{C_{\mathrm{p}}}+R_{2}+R_{3}-\frac{R_{1}}{2}\right) \int s^{2}+\frac{R_{1}}{2} \int z^{2}+\frac{\mu_{6}}{C_{\mathrm{p}}|\Omega|}\left(\int s\right)^{2} .
\end{gathered}
$$

We cannot impose physically reasonable conditions analogous to those used in previous sections that allowed us to ignore the terms $\left(\int y\right)^{2}$ and $\left(\int s\right)^{2}$ that arise from use of the Poincare inequality. These additional terms are obviously non-negative, so at present, we will treat them as we treat the perturbations variables $u^{2}-s^{2}$. That is, we will find sufficient conditions on the various parameters such that

$$
\frac{\mathrm{d}}{\mathrm{d} t}\left(\int y\right)^{2}<0 \text { and } \frac{\mathrm{d}}{\mathrm{d} t}\left(\int s\right)^{2}<0 .
$$

We integrate (11) and (12) over $\Omega$ then multiply by $\left(\int y\right)$ and $\left(\int s\right)$, respectively. Making use of the fact that both $y$ and $s$ satisfy homogeneous Neumann boundary conditions, and using the Cauchy-Schwarz inequality on terms of the form $\left(\int z\right)^{2}$ we obtain

$$
\frac{1}{2} \frac{\mathrm{d}}{\mathrm{d} t}\left(\int y\right)^{2} \leq-\left(Q_{3}-\frac{Q_{2}+Q_{5}}{2}\right)\left(\int y\right)^{2}+\frac{Q_{2}|\Omega|}{2} \int z^{2}+\frac{Q_{5}}{2}\left(\int s\right)^{2}
$$

and

$$
\frac{1}{2} \frac{\mathrm{d}}{\mathrm{d} t}\left(\int s\right)^{2} \leq-\left(R_{2}+R_{3}-\frac{R_{1}}{2}\right)\left(\int s\right)^{2}+\frac{R_{1}|\Omega|}{2} \int z^{2} .
$$

Finally, we can consider the variable $z$. Since we can allow $\alpha_{4}$ to have either sign, there are two cases. If $\alpha_{4}>0$, we have reverse transport. In the absence of medical intervention, this is the less likely case as LDL molecules are typically trapped in the arterial wall. We could ignore this case; however, it is of interest to see what the stabilizing effect is and how it balances with other parameters. 
First, in the case of forward transport, $\alpha_{4}=-\left|\alpha_{4}\right|<0$, we can use the Sobolov inequality and obtain from (35)

$\frac{1}{2} \frac{\mathrm{d}}{\mathrm{d} t} \int z^{2} \leq-\left(\mu_{4}-\left|\alpha_{4}\right| C_{1}\right) \int|\nabla z|^{2}-\left(P_{1}-\left|\alpha_{4}\right| C_{1}-\frac{P_{2}+P_{3}}{2}\right) \int z^{2}+\frac{P_{2}}{2} \int y^{2}+\frac{P_{3}}{2} \int s^{2}$.

The destabilizing effect is readily apparent as we see that the criterion for decay will require $P_{1}$ to increase as $\left|\alpha_{4}\right|$ increases. We will require the condition

$$
\text { [Condition 11.1] } \mu_{4}>\left|\alpha_{4}\right| \text {, if } \alpha_{4}<0 \text {. }
$$

For the reverse transport case $\left(\alpha_{4}>0\right)$, we can consider the expression

$$
-\mu_{4} \int|\nabla z|^{2}-\alpha_{4} \int_{\Gamma_{1}} z^{2}+\phi_{0} \int z^{2}
$$

where

$$
\phi_{0}=\frac{P_{2}+P_{3}+R_{1}+Q_{2}+\left(R_{1}+Q_{2}\right)|\Omega|}{2} .
$$

Application of the Friedrich's inequality to the term $\phi_{0} \int z^{2}$ gives

$$
\begin{aligned}
-\mu_{4} \int|\nabla z|^{2}-\alpha_{4} \int_{\Gamma_{1}} z^{2}+\phi_{0} \int z^{2} \leq & -\left(\mu_{4}-\frac{\phi_{0}}{C_{2}}\right) \int|\nabla z|^{2}-\left(\alpha_{4}-\frac{\phi_{0} C_{3}}{C_{2}}\right) \\
& \times \int_{\Gamma_{1}} z^{2}
\end{aligned}
$$

Here, we will impose

$$
\text { [Condition 11.2] } \mu_{4}>\phi_{0} / C_{2} \text { and } \alpha_{4}>\phi_{0} C_{3} / C_{2} \text { if } \alpha_{4}>0 \text {. }
$$

This latter condition guarantees the existence of a positive constant $\hat{C}$ such that

$$
-\mu_{4} \int|\nabla z|^{2}-\alpha_{4} \int_{\Gamma_{1}} z^{2}+\phi_{0} \int z^{2} \leq-\hat{C} \int z^{2} .
$$

Our primary result for this section is arrived at by summing the inequality (47) [or using (48)] with (41)-(46). Assuming the Conditions 9, 10, hold, and that the appropriate Condition 11.1 or 11.2 holds, we have

$$
\begin{aligned}
& \frac{1}{2} \frac{\mathrm{d}}{\mathrm{d} t} \int\left[u^{2}+v^{2}+w^{2}+z^{2}+y^{2}+s^{2}\right]+\frac{1}{2} \frac{\mathrm{d}}{\mathrm{d} t}\left[\left(\int y\right)+\left(\int s\right)\right] \\
& \quad \leq-\left[C_{u} \int u^{2}+C_{v} \int v^{2}+C_{w} \int w^{2}+C_{z} \int z^{2}+C_{y} \int y^{2}+C_{s} \int s^{2}\right. \\
& \left.\quad+\left(D+G_{1}\right) \int u v+E \int u y+K \int u w+C_{\int y}\left(\int y\right)^{2}+C_{\int s}\left(\int s\right)^{2}\right] .
\end{aligned}
$$


The coefficients on the right-hand side are

$$
\begin{aligned}
C_{u} & =A_{1}-\frac{C_{1}}{2}\left(\alpha_{1}+\frac{\chi \alpha_{3}}{\mu_{3}}\right) \\
C_{v} & =H_{1}-\frac{J+L}{2}, \\
C_{w} & =M_{1}+C\left(\bar{\alpha}, \overline{\mu_{3}}\right) C-\frac{L}{2}, \\
C_{z} & = \begin{cases}P_{1}, & \alpha_{4}>0 \\
P_{1}-\left|\alpha_{4}\right| C_{1}-\frac{P_{2}+P_{3}+R_{1}+Q_{2}}{2}-\frac{\left(R_{1}+Q_{2}\right)|\Omega|}{2}, & \alpha_{4}<0,\end{cases} \\
C_{y} & =\frac{\mu_{5}}{C_{\mathrm{p}}}+Q_{3}-\frac{J+P_{2}+Q_{2}+Q_{5}}{2}, \\
C_{s} & =\frac{\mu_{6}}{C_{\mathrm{p}}}+R_{2}+R_{3}-\frac{P_{3}+R_{1}+Q_{5}}{2}, \\
C_{\int y} & =Q_{3}-\frac{Q_{2}+Q_{5}}{2}, \\
C_{\int s} & =R_{2}+R_{3}-\frac{R_{1}+Q_{5}}{2},
\end{aligned}
$$

Let the vector $\vec{V}=\left(u, v, w, z, y, s, \int y, \int s\right)$, and assume the following condition is met.

[Condition 12] Each of the coefficients $C_{u}, C_{v}, \ldots, C_{\int s}$ are positive.

Define the functional

$$
\mathcal{F}_{3}(\vec{V})=\left(\sum_{i=1}^{6} \int V_{i}^{2}\right)+V_{7}^{2}+V_{8}^{2}
$$

and the parameters

$$
\beta_{u}=\sqrt{\frac{1}{3} C_{u}}, \quad \beta_{v}=\sqrt{C_{v}}, \quad \beta_{w}=\sqrt{C_{w}} \text { and } \beta_{y}=\sqrt{C_{y}} .
$$

Finally, define

$$
\begin{aligned}
\mathcal{F}_{4}(\vec{V})= & \int \frac{1}{2}\left(\beta_{u} u+\beta_{v} v\right)^{2}+\frac{1}{2}\left(\beta_{u} u+\beta_{w} w\right)^{2}+\frac{1}{2}\left(\beta_{u} u+\beta_{y} y\right)^{2} \\
& +M\left(z^{2}+s^{2}+\left(\int y\right)^{2}+\left(\int s\right)^{2}\right)
\end{aligned}
$$

where $M=\min \left\{C_{z}, C_{s}, C_{\int y}, C_{\int s}\right\}$.

THEOREM 2. The equilibrium solution $\left(\mathcal{I}_{\mathrm{e}}, \mathcal{D}_{\mathrm{e}}, \mathcal{C}_{\mathrm{e}}, \mathcal{L}_{\mathrm{e}}, \mathcal{L}_{\text {oxe }}, \mathcal{R}_{\mathrm{e}}\right)$ of (1)-(6) subject to the boundary conditions (28) is asymptotically stable provided Conditions 9-12 hold and if

$$
\beta_{u} \beta_{v} \geq D+G_{1}, \quad \beta_{u} \beta_{w} \geq K \text { and } \beta_{u} \beta_{y} \geq E .
$$


If all hypotheses in Theorem 2 are satisfied, then

$$
\frac{\mathrm{d}}{\mathrm{d} t} \mathcal{F}_{3}(\vec{V}) \leq-\mathcal{F}_{4}(\vec{V})
$$

establishing asymptotic stability for this case analogous to (27) for the previously considered equations.

The parameters $\bar{\mu}_{1}$ and $\bar{\mu}_{3}$ appearing in Conditions 9 and 10, respectively, are positive when the competing effects of diffusion and chemotaxis are such that diffusion dominates. Dominance of diffusion in such systems is again well known to be stabilizing. A comparison of $\bar{\mu}_{1}$ with the parameter $C_{\nabla u}$ from Section 3.1 shows that the inclusion of boundary transport of immune cells due to chemotaxis places a stronger burden on immune cell motility for stabilization. We have

$$
\bar{\mu}_{1}=\mu_{1}-C_{1}\left(\alpha_{1}+\frac{\chi \alpha_{3}}{\mu_{3}}\right)-\frac{\chi}{2} \quad \text { and } \quad C_{\nabla u}=\frac{1}{2}\left(\mu_{1}-\frac{\chi}{2}\right) .
$$

The diffusive capability of immune cells must overcome chemotaxis across the boundary governed by parameters $\alpha_{1}$ and $\alpha_{3}$ - in addition to the interior of the domain. The more likely of the two versions of Condition 11, is the case $\alpha_{4}<0$ since forward transport of LDL molecules and subsequence trapping of such molecules is what is observed. The diffusion parameter $\mu_{4}$ is non-negative; here, Condition 11.1 imposes a lower bound on this value for stability. It means that diffusion must dominate the influx due to haptotaxis at the endothelial layer and high serum LDL levels. Finally, Condition 12 represents a sufficient relationship between various stabilizing and destabilizing factors. Of interest here is the two coefficients $C_{\int y}$ and $C_{\int s}$ unique to the case allowing for boundary transport. The parameters $Q_{2}, Q_{5}$ and $R_{1}$ are oxidation rates with respect to the concentrations of LDL and free-radicals. Parameters $Q_{3}$ and $R_{3}$ are proportional to the anti-oxidant concentration. Positivity of $C_{\int y}$ and $C_{\int s}$ can thus be interpreted as requiring the anti-oxident level to be large as compared to the oxidation reaction rates, which is intuitive as a stability criterion.

\section{Conclusion}

Herein, we have extended the methodology introduced in [9] to study atherogenesis as an inflammatory instability. As before, we are able to obtain physically reasonable stability criterion given, our model of the disease process. Of note at present is the inclusion of the previously neglected interactions involving LDL cholesterol, oxygen and anti-oxidant species. In particular, we see that increasing the anti-oxidant levels in the system, in conjunction with any action that increases diffusivity in the domain has the expected effect of mitigating disease. Moreover, we obtain particular inequalities that can be considered as data becomes available. For example, we found that we require (closed boundary case)

$$
\left(1-\frac{b_{4}}{2}\right) Q_{3}+\mu_{5} C_{\mathrm{p}}>\frac{Q_{2}+Q_{5}+E+J}{2}+\frac{E}{2 D}+\frac{J}{2 G_{1}},
$$

which gives a specific relationship between the magnitude of anti-oxidant reaction $\left(\left(1-b_{4} / 2\right) Q_{3}\right)$, diffusion $\left(\mu_{5}\right)$ and healthy immune function $\left(D\right.$ and $\left.G_{1}\right)$ as compared to the total oxidation rate $\left(Q_{2}+Q_{5}\right)$ and foam cell production $(E+J)$.

The final result suggests that any intervention that can minimize - or to an even larger extent, reverse - the influx of LDL into the intima will provide the greatest degree of stability. What is more, from the Condition 11.2 (reverse transport), and perhaps more 
importantly from the coefficient $C_{z}$ in Section 4 corresponding to transport of LDL into the intima (forward transport), the size of the domain plays a role in stability. Here, we see that the smaller the domain (i.e. $|\Omega|$ ) the more stable the system. If the geometry is known, an explicit stability condition on the parameters can be obtained relative to the shape and thickness of the intimal layer.

Herein, we were also able to adapt the method presented in [9] to the more realistic inclusion of the effects of boundary transport. This is possible as we avoid the mathematical intractability that can occur when using spectral theory. By using Sobolov embedding, we are further able to obtain stronger results. For example, we are able to show that in some cases the gradients of the perturbations, in addition to the perturbations themselves vanish asymptotically.

A number of assumption were made within the current study such as the constant rate of free-radical production and the constant level of anti-oxidant concentration among others. More complex dynamics for additional species such as free radicals, anti-oxidants, and highdensity lipoproteins (HDL) can reasonably be included. Cobbold Sherratt and Maxwell, in their study of LDL oxidation [3], do include and analyse the effect of the presence of HDL known to mitigate LDL oxidation. Expansion of the governing equations to account for additional processes is one topic of ongoing investigation. The method of analysis is flexible and lends itself to such extensions. Additional numerical studies of the various parameters that we obtain herein may further illuminate the relationship between competing reactions such as rates of LDL oxidation as compared to the rate of uptake of oxidized LDL by macrophages, or the rate of production of chemoattractant as compared to its natural degradation and diffusivity. As in [9], we propose that the methodology presented here is also adaptable to systems other than those considered presently.

\section{Acknowledgements}

This publication is based on work supported in part by Award No. KUS-C1-016-04, made by King Abdullah University of Science and Technology (KAUST).

\section{Notes}

1. We note here that 'debris' is applied in perhaps a non-conventional way. We do not mean to say that the material we are calling debris is an inert byproduct of some other process that plays no role other than occupying space. In fact, as will be seen in the mathematical model to follow, debris - more precisely lesion debris - is an active species participating in the inflammatory feedback loop. Having used the term 'debris' for this species in the previous works [7-9], we will herein follow this convention.

2. In the classical Keller-Segal model for dictyostelium discoideum, for example, $\chi(\mathcal{I}, \mathcal{C})$ has the form $\chi(\mathcal{I}, \mathcal{C})=\mathcal{I} / \mathcal{C}[11]$. At present, there is no need to specify a particular form for $\chi(\mathcal{I}, \mathcal{C})$.

3. How to model the various 'reaction' terms in the governing equations is a significant issue. Classical 'mass action' kinetics is adopted here mostly for illustrative purposes. However, the coefficients are not assumed to be constants; rather they are functions of the reacting or interacting species and can capture saturation effects in the reaction rates, for example. For the stability results considered here, it is not necessary to adopt specific forms for the reaction rates. However, one must specify values for the reaction rates at a healthy state in order to apply the stability results to any particular concrete setting. Naturally, specific forms for the coefficients are required in order to prove the existence of or find healthy (equilibrium) states. In particular, this is done below for the special case of constant reaction coefficients.

4. We are incorporating a simplified version of the model of Cobbald Sherratt and Maxwell for LDL modification which presently supresses the multiple stage oxidation process found in [3]. Our generalized native LDL species can be considered as a sum of all LDL that is not fully chemically modified. The anti-oxidant reaction likewise here appears as a single reaction. 
5. Free radical production is considered here to be a metabolic byproduct which for present purposes will be assumed to be constant.

6. A number of conditions, including this one imposed on $\Lambda$, will be made. The physical interpretations will be discussed following the statement of our obtained results.

7. See Appendices A and B for an argument that (27) implies asymptotic stability in the sense defined previously.

8. In the case of an annulus of inner and outer radii $r_{\mathrm{I}}$ and $r_{\mathrm{O}}$, respectively,

$$
C_{1}=\frac{4 \ln \left(r_{\mathrm{O}} / r_{\mathrm{I}}\right)}{r_{\mathrm{O}}^{2}-r_{\mathrm{I}}^{2}}, \quad C_{2}=\left(\left(r_{\mathrm{O}}^{2}-r_{\mathrm{I}}^{2}\right) \ln \left(r_{\mathrm{O}} / r_{\mathrm{I}}\right)\right)^{-1} \text { and } C_{3}=1 / \ln \left(r_{\mathrm{O}} / r_{\mathrm{I}}\right)
$$

\section{References}

[1] G. Acosta and R.G. Durán, An optimal Poincaré inequality in $L^{1}$ for convex domains, Proc. Am. Math. Soc. 132(1) (2003), pp. 195-202.

[2] C.G. Caro, J.M. Fitz-Gerald, and M.F. Schroter, Atheroma and arterial wall shear: observation, correlation and proposal of a shear dependent mass transfer mechanism for atherogenesis, Proc. R. Soc. Lond. B 177 (1971), pp. 109-133.

[3] C.A. Cobbold, J.A. Sherratt, and S.J.R. Maxwell, Lipoprotein oxidation and its significance for atherosclerosis: a mathematical approach, Bull. Math. Biol. 64 (2002), pp. 65-95.

[4] M.A. Creager, ed., Atlas of Vascular Disease, 2nd ed., Current Medicine, Inc., Philadelphia, 2003.

[5] J. Fan and T. Watanabe, Inflammatory reactions in the pathogenesis of atherosclerosis, JAT 10(2) (2003), pp. 63-71.

[6] J.L. Goldstein, Y.K. Ho, S.K. Basu, and M.S. Brown, Binding site on macrophages that mediates uptake and degradation of acetylated low density lipoproteins, producing massive cholesterol deposition, Proc. Natl Acad. Sci. USA 76 (1977), pp. 333-337.

[7] A.I. Ibragimov, C.J. McNeal, L.R. Ritter, and J.R. Walton, Stability analysis of a model of atherogenesis: an energy estimate approach, J. Comp. Math. Methods Med. 9(2) (2008), pp. 121-142.

[8] A.I. Ibragimov, C.J. McNeal, L.R. Ritter, and J.R. Walton, A dynamic model of atherogenesis as an inflammatory response, Dyn. Contin. Discrete Impuls. Syst. Ser. A 14(S2) (2007), pp. $185-189$.

[9] A.I. Ibragimov, C.J. McNeal, L.R. Ritter, and J.R. Walton, A mathematical model of atherogenesis as an inflammatory response, Math. Med. Biol. 22 (2005), pp. 305-333.

[10] G.E. Jones, Cellular signaling in macrophage migration and chemotaxis, J. Leuko. Biol. 68 (2000), pp. 593-602.

[11] E.F. Keller and L.A. Segel, Model for chemotaxis, J. Theor. Biol. 30 (1971), pp. 235-248.

[12] M.J. Lever and M.T. Jay, Convective and diffusive transport of plasma proteins across the walls of large blood vessels, Front. Med. Biol. Eng. 5 (1993), pp. 45-50.

[13] G. Pasterkamp, A.H. Schoneveld, W. van Wolferen, B. Hillen, R.J.G. Clarijs, C.C. Haudenschild, and $\mathrm{C}$. Borst, The impact of atherosclerotic arterial remodeling on percentage of luminal stenosis varies widely within the arterial system: a postmortem study, Arterioscle. Thromb. Vasc. Biol. 17 (1997), pp. 3057-3063.

[14] A. Quarteroni, Modeling the cardiovascular system: a mathematical challenge, in Mathematics Unlimited, B. Engquist and W. Schmid, eds., Springer-Verlag, Berlin, 2001, pp. 961-972.

[15] R. Ross, Atherosclerosis - an inflammatory disease, New Engl. J. Med. 340(2) (1999), pp. $115-126$.

[16] R. Ross, Cell biology of atherosclerosis, Annu. Rev. Physiol. 57 (1995), pp. 791-804.

[17] P.W.F. Wilson, ed., Atlas of Atherosclerosis: Risk Factors and Treatments, 2nd ed., Current Medicine, Inc., London, 2000.

\section{Appendix A}

Define $\Phi_{1}(t)=\hat{\Phi}_{1}(\vec{\xi}(t))=\vec{\xi}(t) \cdot \mathbf{A}_{1} \vec{\xi}(t)$ and $\Phi_{2}(t)=\hat{\Phi}_{2}(\vec{\xi}(t))=\vec{\xi}(t) \cdot \mathbf{A}_{2} \vec{\xi}(t)$ for $\vec{\xi}$ in $\mathbb{R}^{n}$, with $\mathbf{A}_{1}$ and $\mathbf{A}_{2}$ positive definite matrices. 
Lemma. Suppose

$$
\frac{\mathrm{d}}{\mathrm{d} t} \Phi_{1}(t) \leq-\Phi_{2}(t), \quad \text { for } t>t_{0}
$$

and $\Phi_{1}\left(t_{0}\right)>0$. Then

$$
\lim _{t \rightarrow \infty} \vec{\xi}(t)=\overrightarrow{0}
$$

Proof. First note that $\Phi_{1}(t) \rightarrow 0$ if and only if $\vec{\xi}(t) \rightarrow \overrightarrow{0}$, and that $\Phi_{1}$ is both non-negative and nonincreasing. Thus $\lim _{t \rightarrow 0} \Phi(t)$ exists and is either zero or a finite number. Suppose $\lim _{t \rightarrow 0} \Phi(t)>0$. It follows that there exists $a_{1}>0$ such that $\|x \vec{i}(t)\|>a_{1}$ for all $t>t_{0}$. It then follows that there exists $a_{2}>0$ such that $\Phi_{2}(t)>a_{2}$, for all $t>t_{0}$, and hence that

$$
\frac{\mathrm{d}}{\mathrm{d} t} \Phi_{1}(t) \leq-a_{2}, \quad \text { for } t>t_{0} .
$$

But it then follows that there exists $T>t_{0}$ such that $\Phi(t)<0$ for all $t>T$, in contradiction to the non-negativity of $\Phi_{1}$. We can thus conclude that $\Phi(t) \rightarrow 0$ and hence $\lim _{t \rightarrow 0} \vec{\xi}(t)=\overrightarrow{0}$.

\section{Appendix B: $\mathrm{d} / \mathrm{d} t\left(\mathcal{F}_{1}(t)\right) \leq-M \mathcal{F}_{2}(t)$ implies asymptotic stability}

If $\mathcal{F}_{1}\left(t_{0}\right)=0$ for some $t_{0}$, then $\mathcal{F}_{1} \equiv 0$ since $\mathcal{F}_{1}$ is monotone and non-negative. Otherwise, suppose that there exists a trajectory $\mathbf{Y}$ exceeding some point $\mathbf{Y}_{0}$ at $t=0$ such that for for all $t>0 \mathbf{Y}$ remains outside of a ball of radius $R_{0}>0$. Then at least one of the 10 components of $\mathbf{Y}$ exceeds $R_{0} / 10$.

We note that the left- and right-hand sides of the inequality differ only with respect to $Y_{1}, Y_{2}$ and $Y_{3}$. It should thus be considered what happens in the case that one of these components is larger than $R_{0} / 10>0$. If $Y_{1}>R_{0} / 10$, then as a result of the Poincaré inequality, there exists $c>0$ such that $Y_{9}>c R_{0} / 10$. Similarly, if $Y_{3}>R_{0} / 10$, there exists $c>0$ such that $Y_{10}>c R_{0} / 10$. Finally, if $Y_{2}>R_{0} / 10$ and $Y_{1}=0$, then there exists some $c>0$ such that $Y_{7}>c R_{0} / 10$.

We can conclude then that there exist positive constants $a$ and $T$ such that for $t>T$

$$
\mathcal{F}_{2}(t) \geq a,
$$

where $a$ depends on the coefficients of the linear system through $M$ and depends on $R_{0}$ through $\mathbf{Y}$. But then

$$
\frac{\mathrm{d}}{\mathrm{d} t} \mathcal{F}_{1}(t) \leq-a
$$

so that

$$
\mathcal{F}_{1}(t) \leq-a t+\mathcal{F}_{1}(0)
$$

But, for sufficiently large $t$ this contradicts the non-negativity of $\mathcal{F}_{1}$. So $\mathcal{F}_{1}$ must vanish at infinity. 


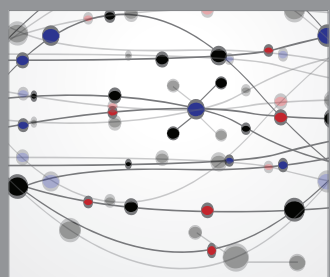

The Scientific World Journal
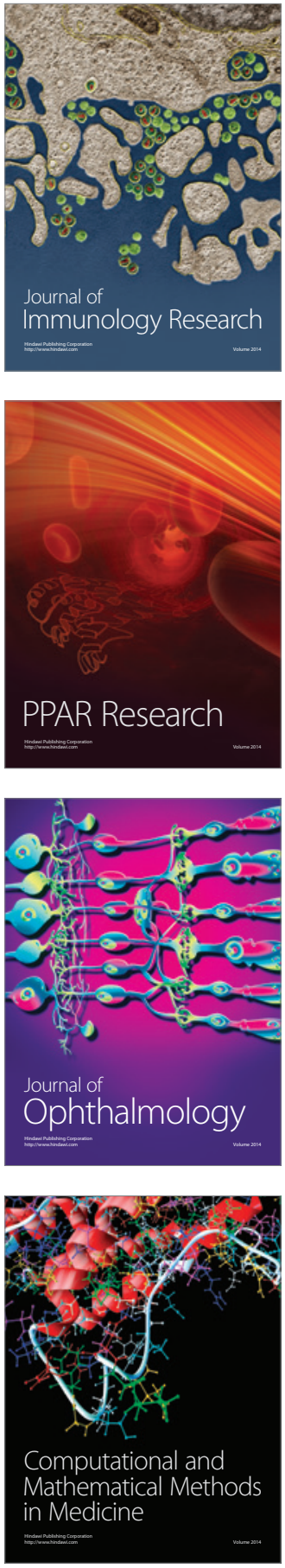

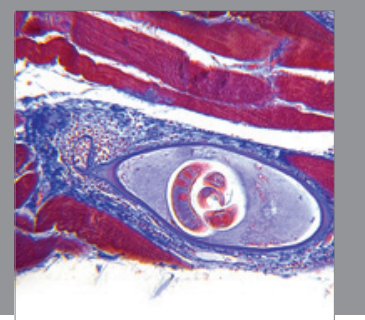

Gastroenterology

Research and Practice
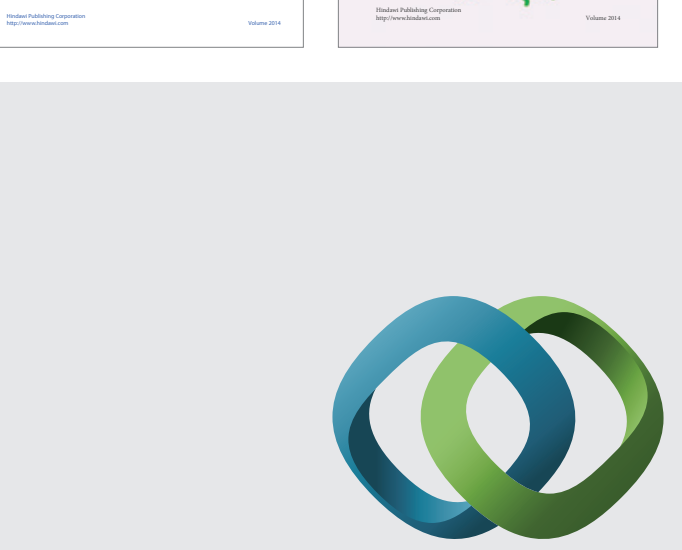

\section{Hindawi}

Submit your manuscripts at

http://www.hindawi.com
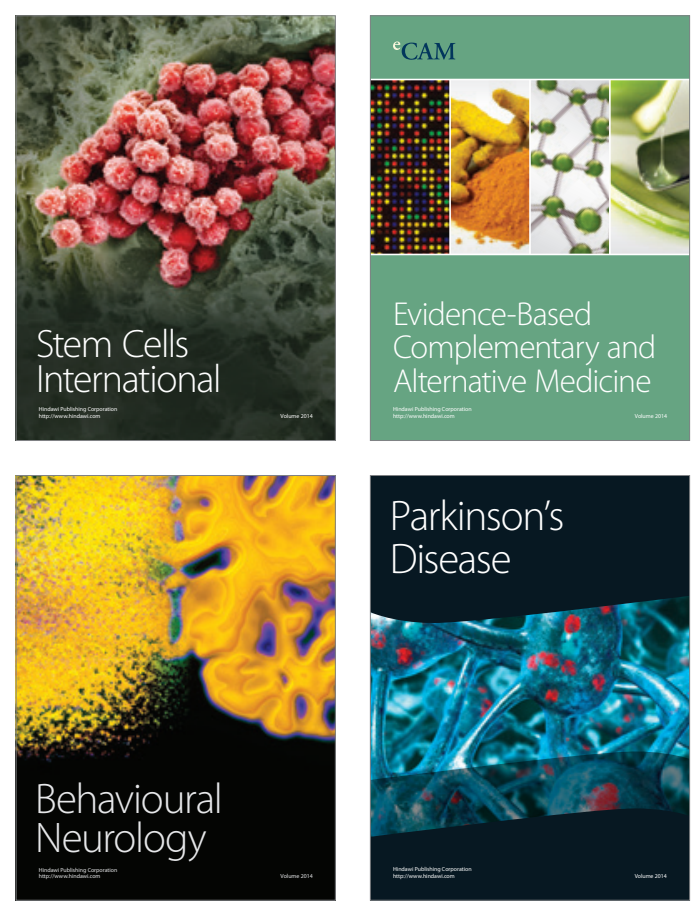

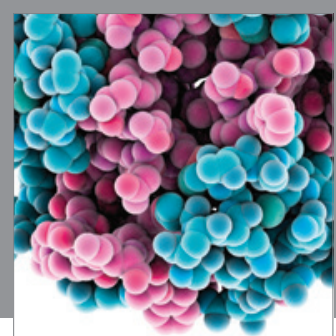

Journal of
Diabetes Research

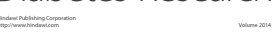

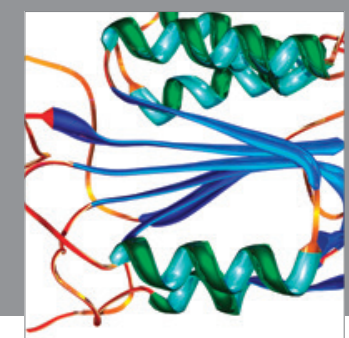

Disease Markers
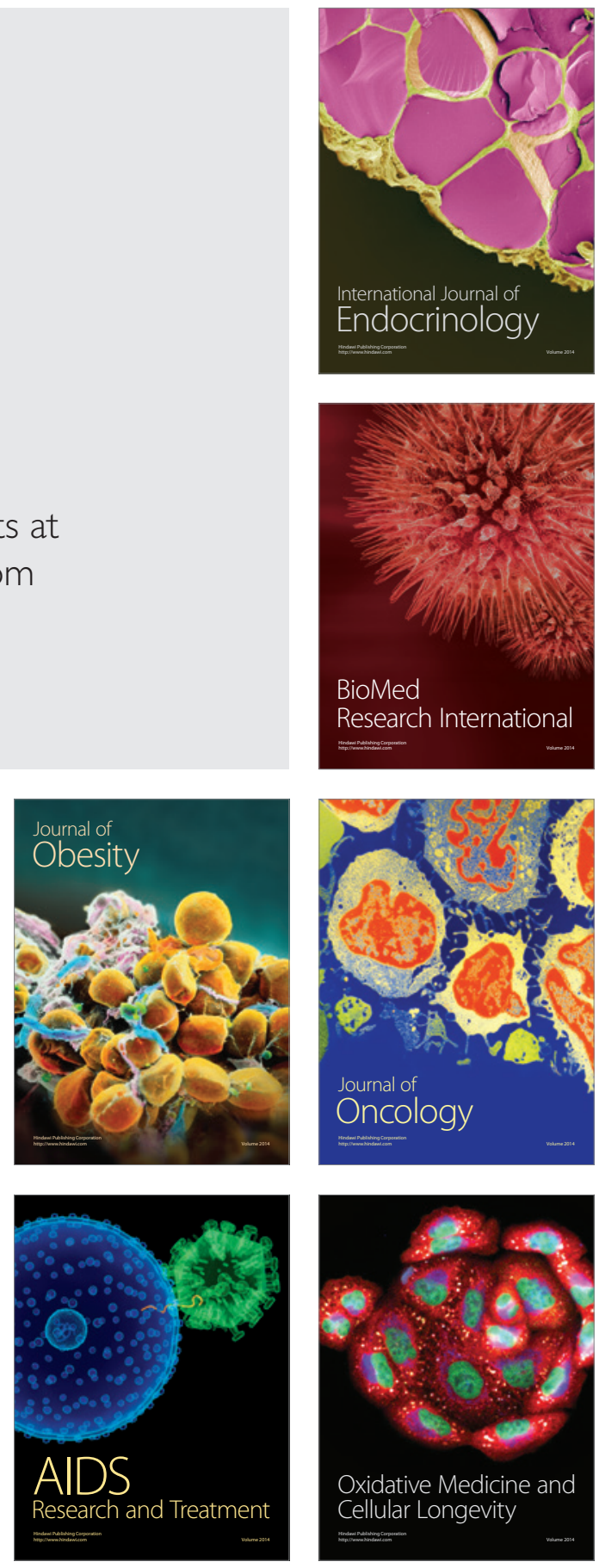